(C) [2008] IEEE. Reprinted, with permission, from [Zhan,g Zhengzhi; Kodagoda, Sarath; Ruiz, David; Katupitiya, J; Dissanayake, Gamini. Classification of Bidens in wheat farms. Mechatronics and Machine Vision in Practice, 2008. M2VIP 2008. 15th International Conference ]. This material is posted here with permission of the IEEE. Such permission of the IEEE does not in any way imply IEEE endorsement of any of the University of Technology, Sydney's products or services. Internal or personal use of this material is permitted. However, permission to reprint/republish this material for advertising or promotional purposes or for creating new collective works for resale or redistribution must be obtained from the IEEE by writing to pubspermissions@ieee.org. By choosing to view this document, you agree to all provisions of the copyright laws protecting it 


\title{
Classification of Bidens in Wheat Farms
}

\author{
Z. Zhang ${ }^{\text {a }}$, S. Kodagoda ${ }^{\text {a }}$, D. Ruiz ${ }^{\mathrm{b}}$, J. Katupitiya ${ }^{\mathrm{c}}$, G. Dissanayake ${ }^{\mathrm{a}}$ \\ ${ }^{a}$ ARC Centre of Excellence for Autonomous Systems (CAS), University of Technology, Sydney,AUSTRALIA \\ ${ }^{\mathrm{b}}$ Australian Centre for Precision Agriculture (ACPA), University of Sydney, Sydney, AUSTRALIA \\ ${ }^{c}$ ARC Centre of Excellence for Autonomous Systems (CAS), University of New South Wales,AUSTRALIA
}

\begin{abstract}
Bidens pilosa $L$ (commonly known as cobbler's peg) is an annual broad leaf weed widely distributed in tropical and subtropical regions of the world and is reported to be a weed of 31 crops including wheat. Automatic detection of Bidens in wheat farms is a nontrivial problem due to their similarity in color and presence of occlusions. This paper proposes a methodology which could be used to discriminate Bidens from wheat to be used in operations such as autonomous weed destruction. A spectrometer is used to analyze the optical properties of Bidens and wheat leaves while achieving high classification results. However, due to the practical constraints of using spectrometers, a color camera based technique is proposed. It is shown that the color based segmentation followed by shape based validation algorithm gives rise to high detection rates with lower false detections. We have experimentally evaluated the algorithm with Bidens detection rate of $80 \%$ and a $10 \%$ false alarm rate.
\end{abstract}

\section{INTRODUCTION}

There are a number of field operations that can be executed by autonomous vehicles, giving more benefits than conventional man-guided machines [1]. These vehicles would be able to work unattended over long periods of time, carrying out useful tasks such as cultivation and seeding, weed control, soil scouting, application of fertilizers, irrigation, and harvesting. Automatic guidance may avoid over-application of chemicals and fertilizers, reducing environmental impact [2]. As weed populations have been found to be distributed heterogeneously in time and space within agricultural fields, weed control systems based on vision have been developed to spray specifically the weed infested areas in real-time, reducing treatment costs as well as herbicide loading to the environment [3-4].

The authors are collaborating with Eaton et al [5] to realize an autonomous weed control system based on CASPA weeder shown in Figure 1. The CASPA weeder has the capabilities of remote control, joystick based control, sensing and data logging. It can be programmed for autonomous navigation. It has accurate GPS localization system and weighs $89 \mathrm{~kg}$ with approximate size of $800 \mathrm{~L}$ x $550 \mathrm{~W}$ x $400 \mathrm{H}(\mathrm{mm})$. It is planned to implement a fully insulated and isolated electrocution cradle extending out at the back of the robot to be used to destroy the weeds. It will have five independent electrodes at $20 \mathrm{kV}$ covering $250 \mathrm{~mm}$ width and a spiked caster wheel as the ground electrode. The authors' main contribution to this project is to synthesize a low cost, real time crop-weed classification algorithm.

Classification methods reported in the literature fall into two categories: spectral based and computer vision based classification. Spectral based technology relies on the difference between the spectral response of each plant species. Jurado et al [6] used a NIRS monochromator to measure plants' spectral reflectance in a lab environment. The spectral reflectance data was then analyzed statistically. They had shown that the spectral difference in the band of $750 \mathrm{~nm}$ to $950 \mathrm{~nm}$ was suitable for discriminating sunflower and wheat stubbles. However, it was also reported that the results were affected by larger variance in the response of a particular type of plant species. Borregaard et al [7] adopted a line scan spectral device to implement on-site weed detection. In their work, a line scan spectrometer was mounted pointing down observing plants. The line scanning spectrometers provided additional spatial and textural information to that of normal spectrometers with a spot foot print. Classification methods such as linear discriminant analysis (LDA), quadratic discriminant analysis (QDA), principal component analysis (PCA), and partial least-squares regression (PLS) were used to classify the spectral data. However, the accuracy of Borregaard's algorithm was as low as $60 \%$ in some cases.

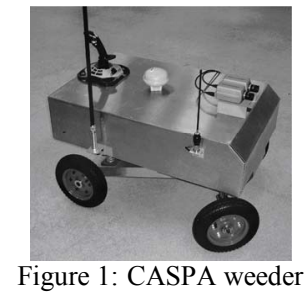

Eddy et al [9] used a hyper-spectral camera with a resolution of $640 \times 480$ pixels. Each pixel of this camera had a spectral resolution of $10 \mathrm{~nm}$ within the spectral band of $400 \mathrm{~nm}$ to $1000 \mathrm{~nm}$. A feed forward neural network was trained to classify the spectral data, and a detection rate of $88 \%-95 \%$ was achieved. The hyper-spectral camera could capture more information than line scan spectrometers, however, the high cost prevented its use in practical weed detection systems.

Computer vision based techniques have also been extensively exploited for crop-weed discrimination. A color image is rich in information providing cues such as color, texture, shape, etc. Perez et al [10] chose color and shape as appropriate visual cues. The cues were then analyzed and classified using three algorithms: heuristical approach, Bayes classification and k-nearest Neighbor classification. Detection rate of $71 \%$ to $89 \%$ was achieved. Aitkenhead et al [11] and Hemming et al [12] proposed to use shape features and 
achieved detection rate ranging from $50 \%$ to $90 \%$. Large variation of the detection performance indicated that the accuracy of the shape parameter calculation was not robust. This can be due to occlusions introduced by nearby leaves.

In this paper, we pay particular attention to classification of Bidens pilosa L (commonly known as cobbler's peg) in wheat crops. Bidens is an annual broad leaf weed widely distributed in tropical and subtropical regions of the world and is reported to be a weed that created problems in farming of 31 crops [13]. Wheat and Bidens have a substantial overlap in the visual spectrum, which causes color alone.segmentation is erroneous. Therefore, in this paper we have proposed a color based Bidens detection followed by a deformable template based shape validation for improved weed detection results. Section II of this paper discusses the spectral analysis of Bidens and wheat. Then in section III, Color based Bidens detection is demonstrated. In section IV, deformable template based shape validation algorithm is presented. Experimental results are presented in Section V. Section VI concludes the paper.

\section{SPECTRAL ANALYSIS OF PLANT LEAF}

\section{A. Spectral analysis}

It is a well-known fact that optical properties of plants can be used to monitor plant growth, disease, crop status, water and nutrient content, and plant type discrimination. Spectral response of leaves depend on pigments in the UV and visible wavelengths, and on chemical composition in the NIR range. Numerous leaf tissue structures also have a significant influence on the spectral properties. Leaf pigments, which contains Chlorophyll absorbs large amount of light in the UV band, blue $(450 \mathrm{~nm})$ and red $(680 \mathrm{~nm})$ part of the spectrum, whilst slightly lower absorption in the green $(550 \mathrm{~nm})$. The leaf pigments do not have a good NIR light absorption property, hence the leaves specularly reflect or diffuse large amount of incident radiation. The surface conditions of leaves, such as presence of hair, can affect the specular reflections. The diffuse reflection is due to part of the transmitted radiation, which is affected by the leaf internal structure and air cavities. Specular and diffuse reflections can be effectively utilized for discrimination of plant species.

Figure 2 shows the spectral response of Bidens and wheat leaves under laboratory conditions. The plants were grown in trays and spectral responses were measured with artificial lighting using an Oceanoptic spectrometer [14]. The steep slope in the response seen between $700 \mathrm{~nm}$ to $750 \mathrm{~nm}$, is called the "red edge". It shows the transition from high absorption by chlorophyll in the red to low absorption in the NIR, which generally are used to calculate the "biomass" or "vegetation indices" in remote sensing.

After analyzing large number of data sets, it was noted that the spectral responses within a given plant type could significantly vary causing a straightforward segmentation based on spectral analysis is erroneous. While it can be seen in Figure 2, that the spectral responses of Bidens and wheat leaves were seen to be different in green $(550 \mathrm{~nm})$ part of the spectrum and NIR band (750nm to $950 \mathrm{~nm})$, Figure 3 shows the spectral variations of Bidens and wheat leaves leading to obvious overlapping regions. It is to be noted that the term "Max" in the legend refers to the maximum spectral response received for a particular leave and "Min" refers to that of the minimum in large number of measurements. This variation in spectral response can be due to age of leaves, their orientation, spatial position, plant health, etc.

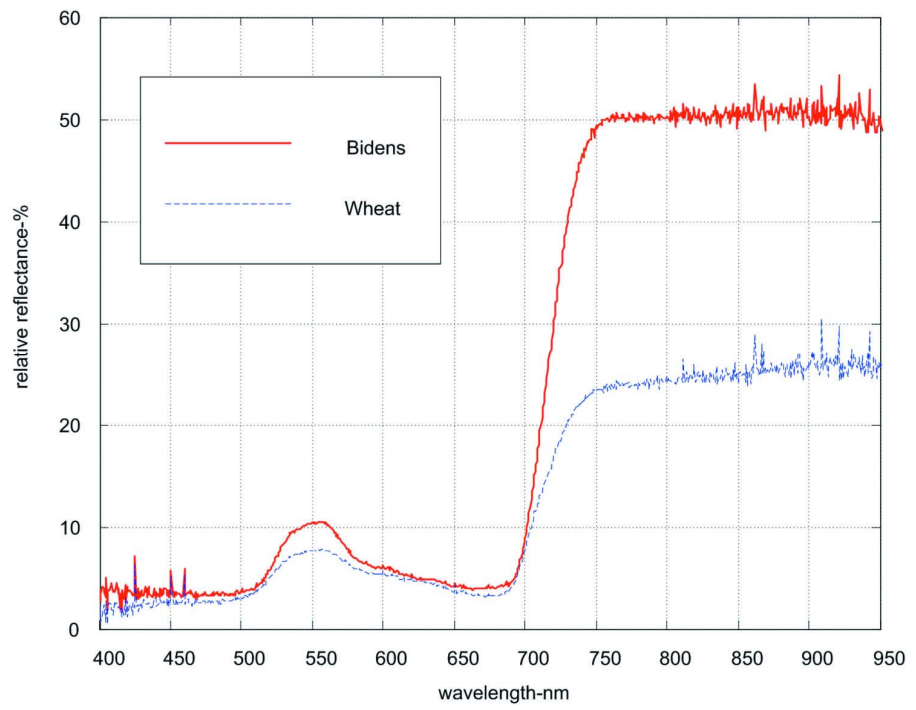

Figure 2: Spectral reflectance

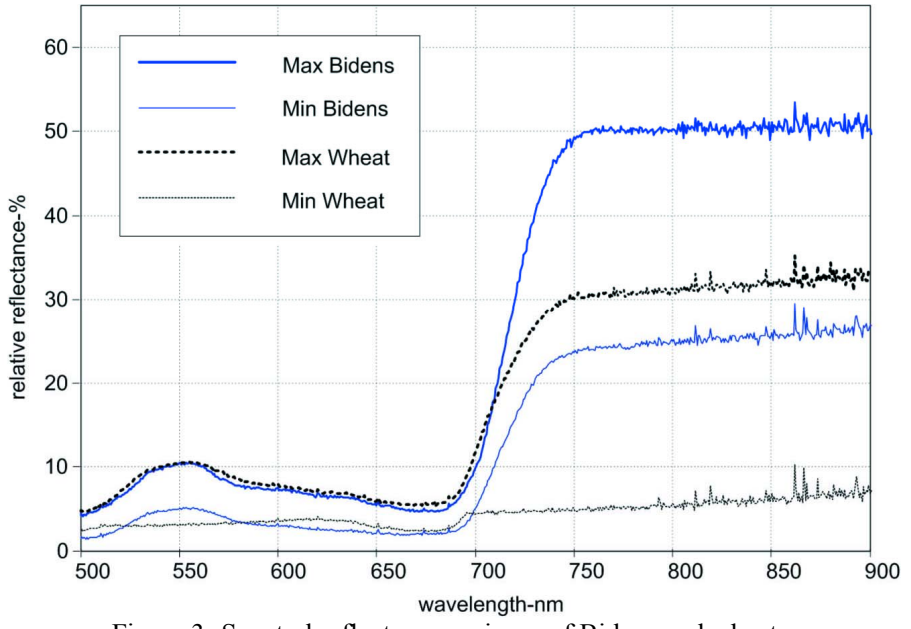

Figure 3: Spectral reflectance variance of Bidens and wheat

\section{B. Classification based on leaf spectral reflectance}

We made an attempt to classify the Bidens and wheat species based on the spectral responses taken from leaves. We captured 25 number of spectral responses of wheat species and 25 number of spectral responses of Bidens species. The spectral range considered was $270 \mathrm{~nm}$ to $1400 \mathrm{~nm}$. The classification was implemented using the Weka [15], a popular machine learning software. We chose NaiveBayes, BayesNet, support vector machine (SVM), and sequential minimal optimization (SMO) as classifiers. Firstly, the entire spectrum was used for the classification, in which, the results are listed 
in Table I. All the classification algorithms provided good results.

TABLE I Classification results based on the entire spectrum

\begin{tabular}{|c|c|c|c|}
\hline $\begin{array}{c}\text { Classification } \\
\text { method }\end{array}$ & Bidens(\%) & wheat(\%) & Time consumed(s) \\
\hline NaiveBayes & 88 & 100 & 0.15 \\
\hline BayesNet & 96 & 100 & 0.47 \\
\hline SVM & 96 & 100 & 75 \\
\hline SMO & 96 & 96 & 0.35 \\
\hline
\end{tabular}

Although classification based on the entire spectrum performed well, there were possible redundant data that could be removed to reduce the computational effort, which is an important consideration in a practical implementation. A typical strategy is to extract informative features and use them for classification. Therefore, we chose the visible spectral band (red, green, blue), near infra-red band(NIR) and 4 vegetation indices [16] derived from data, namely, Vegetation Index(VI), Ratio Vegetation Index(RVI), Transformed Vegetation index(TVI) and red/green ratio. The classification results based on those features (Table II) were comparable with that of using the whole spectrum (Table I), however with a low computational effort.

TABLE II Classification results based on RGB, NIR and vegetation indices

\begin{tabular}{|c|c|c|c|}
\hline $\begin{array}{c}\text { Classification } \\
\text { method }\end{array}$ & Bidens(\%) & wheat(\%) & Time consumed(s) \\
\hline NaiveBayes & 100 & 92 & 0.01 \\
\hline BayesNet & 100 & 92 & 0.01 \\
\hline SVM & 96 & 100 & 0.02 \\
\hline SMO & 100 & 92 & 0.01 \\
\hline
\end{tabular}

Despite the high accuracy of spectrometer based methods, they may not be appropriate solutions for online weed/crop classification due to either their higher costs or practical constraints. One such limitation is the requirement of a reference spectral response at the measuring point for determining the relative reflectance. Another limitation is the inherent small footprint, which requires a sweeping mechanism for obtaining the special information. The spectrometer also needs to be closely located to the measuring surface for accurate readings. Therefore, in this paper we attempt to use a color camera for detecting Bidens among wheat crops. Among all the visual cues, we use color and shape features due to higher discriminative properties.

\section{COLOR BASED WHEAT AND BIDENS DISCRIMINATION}

As discussed in the previous section, color can be used to detect Bidens canopies among wheat. An algorithm based on a learnt color model will be introduced in this section. First, as an offline activity, a small window of Bidens leaf of an incoming image is manually selected (Figure 4) and used to generate the Bidens color model. Then this color model is transformed from RGB color space to HSV color space. Extraction of hue and saturation components from HSV model provides a training sample set. Once the sample set is established, it is possible to synthesize a classification algorithm based on Bradski's et al [17]. Firstly, the incoming image (Figure 5(a)) is processed to eliminate the soil by a simple color based classifier. Then by going through each foreground pixel (Figure 5(b)), the Mahalanobis distance (MD) to the established color model is calculated. Pixels that had MD values less than 1 are assigned a value of 1 and the other pixels are assigned a value of $1 / \mathrm{MD}$. This results in an "image" describing the confidence that a pixel belongs to the established model (Figure 5(c)). The confidence image is then thresholded and morphological operations are performed to improve the connectivity of highly probable pixels while rejecting outliers (Figure 5(d)). The Figure 5(e) shows the final results of the color based Bidens classification of Bidens.

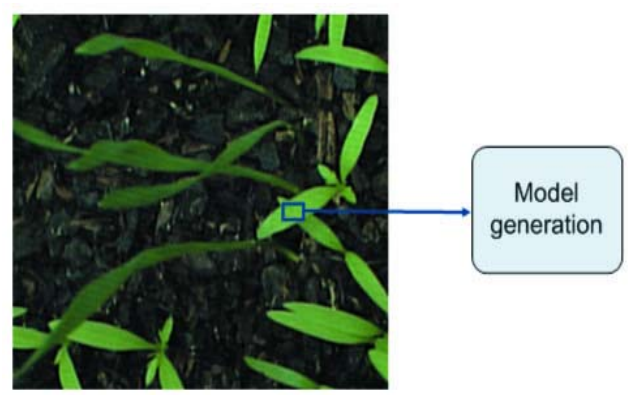

Figure 4: Color model generation

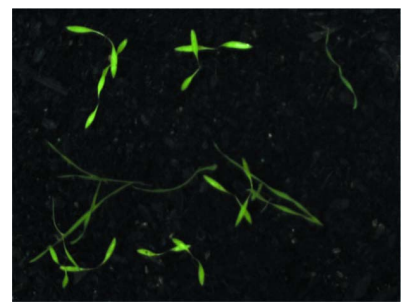

(a) Raw color image

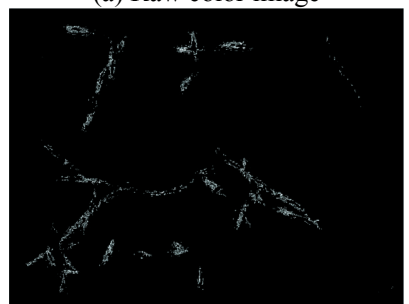

(c) Probabilistic map of Bidens

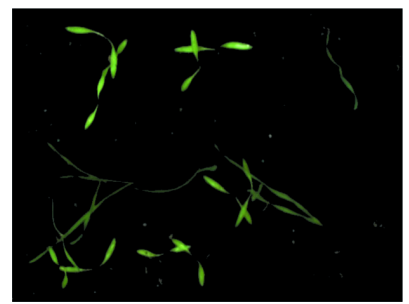

(b) Foreground image

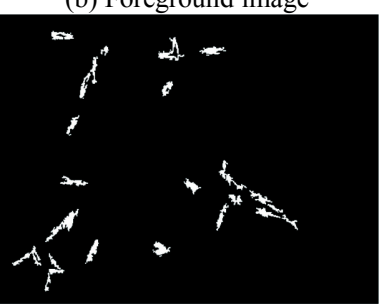

(d) Segmented Bidens

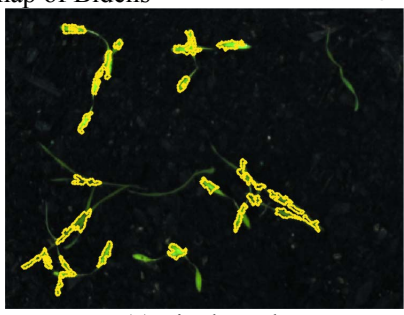

(e) Final result

Figure 5: Snapshots of various stages of Bidens classification 
Figure 6 and Table III show the color based classification results. As can be seen from the Table III, the color based classification provides high Bidens detection rates. However, the false alarm rates are unacceptably high as well. This is due to the similarity in color of some parts of the two plant species. As the main focus of the paper is to detect Bidens which are present in wheat fields and ultimately destroy them, it is important to reduce the false alarm rate to a minimum. For this purpose, we attempted to utilize other visual cues. Next section describes the improvements achieved by incorporating shape validation with the color based detection method.
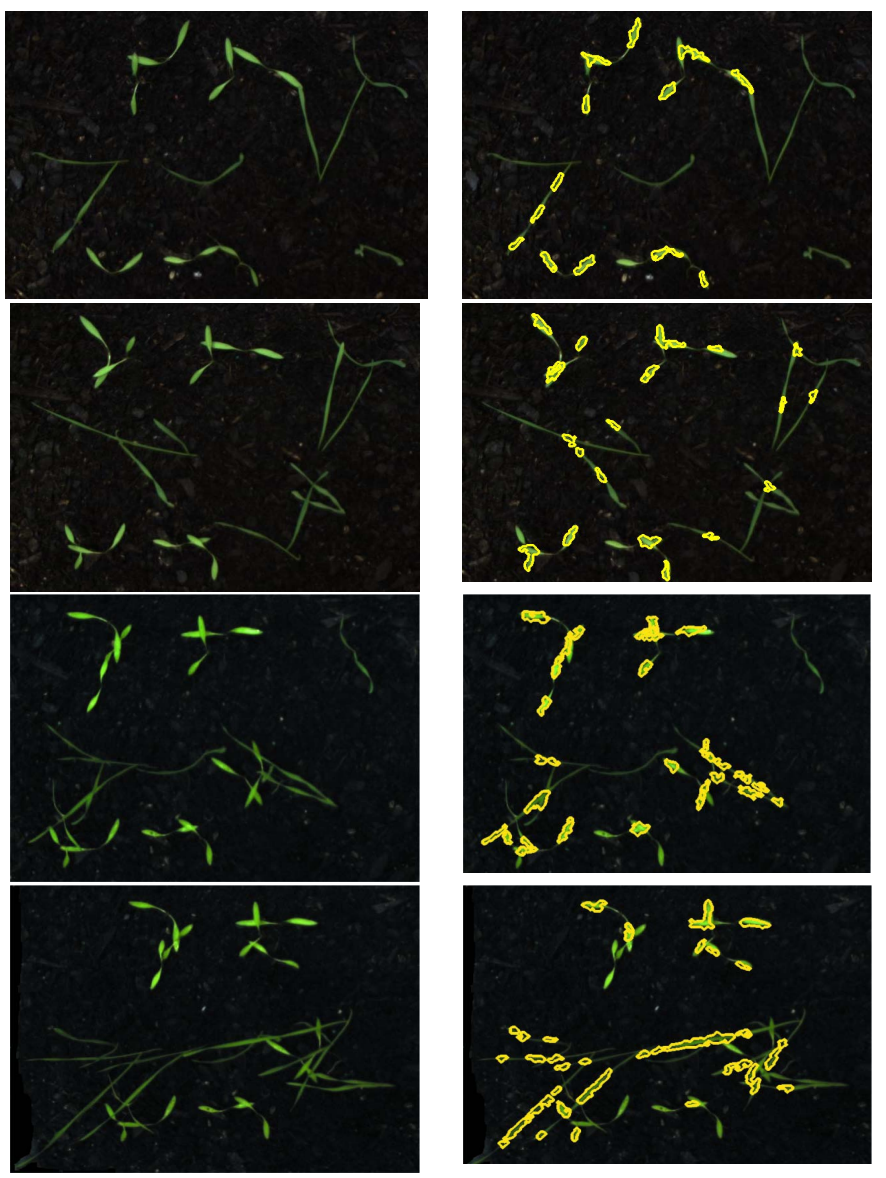

(a) Raw color image

(b) Results

Figure 6: Color based classification results

TABLE III Color based Bidens detection result

\begin{tabular}{|c|c|}
\hline Detection rate & $90.7 \%$ \\
\hline False alarm rate & $35.6 \%$ \\
\hline
\end{tabular}

\section{DEFORMABLE TEMPLATE BASED SHAPE VALIDATION}

For the purpose of reducing false alarms, we propose to introduce an additional shape based validation step after the color based classification. Shapes of Bidens and wheat leaves appear to be significantly different in most cases. Wheat leaves are thin/longer and Bidens leaves are shorter and broad. In this paper, we utilize these differences in shape and a shape model for the Bidens leaves.

Shape based features are widely used in classification. However, the effectiveness of shape features significantly depends on the presence of occlusions. Particularly, in crop/weed classification problem, occlusions cause simple shape parameters to be useless. Therefore, robust shape detection algorithms which can handle occlusions are of importance. There is only hand full of literature on robust shape detection methodologies reported to be used in segmenting leaves. One of them, work by Manh et al [19] seems to be more appropriate to our problem. They built a leaf deformable template model for leaf segmentation. It can cope with slight occlusions. However, the initialization of the template requires an exhaustive search within an angle range between $0 \sim 360$ degrees, which is time consuming. Therefore, we have slightly modified their algorithm for improving computational effort.

The parametric curves of the deformable template are defined by [19],

$$
\begin{aligned}
& x_{s}(i)=a_{x} i^{2}+b_{x} i+c_{x} \\
& y_{s}(i)=a_{y} i^{2}+b_{y} i+c_{y} \\
& f e(i)=\frac{b i}{n\left(1-\frac{i}{n}\right)}
\end{aligned}
$$

where, $i$ is the index of landmark points. $x_{s}$ and $y_{s}$ represent the points on the skeleton model. $a_{x}, b_{x}, c_{x}, a_{y}, b_{y}$, and $c_{y}$ are parameters of the skeleton model. $f e$ is the envelope model. $b$ defines parameters of the envelope model. $n$ is the total number of landmark points.

A flowchart of the deformable template matching algorithm is shown in Figure 8. First the image is binarized based on color to alleviate the background. Then Morphological operations are performed to obtain skeletons (Figure 9(b)). Then by scanning for each tip, a parametric model of Bidens (Figure 7) is initialized for template based matching algorithm (Figure 9(c)). The template growing process is then applied to all the tips that are not previously occupied by another template. The template growing is based on forces acting perpendicular to the parametric curves. Similar colours to Bidens outside parametric curve gives rise to stretching forces where as other colours on the outside produce forces such that the curve shrinks. This lead to deformable template matching as in Figure 9(e). Then the enclosed areas under the parametric curves are set to 1 producing a shape based binarized image (Figure 9(f)). Bidens leaves that were detected based on color (Figure 9(d)) are then corresponded with original color based thresholded image and used to calculate the percentage of overlap with the shape based binary image. The leaves having high percentage of overlap (we assumed it to be above $70 \%$ ) pointed to Bidens whereas wheat leaves had lower values due 
to the elongated nature (Figure 9(g)). Figure 9(h) shows the final result.

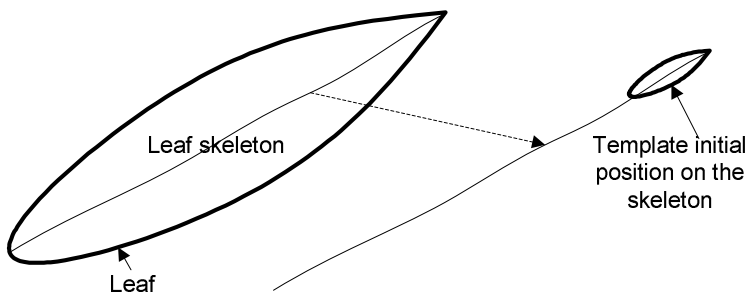

Figure 7: Initialization of deformable template

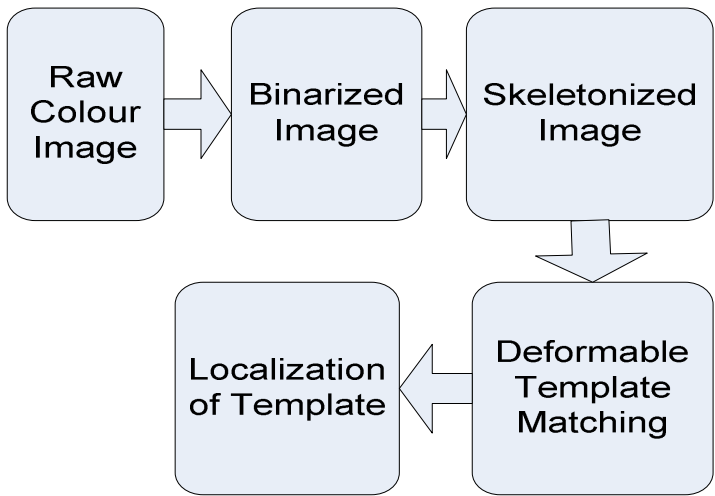

Figure 8: Block diagram of deformable template matching algorithm

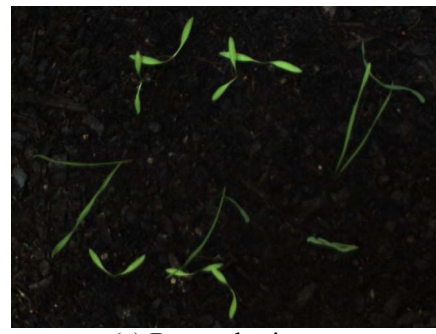

(a) Raw color image

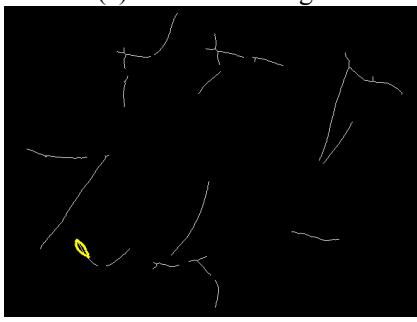

(c) Template initialization

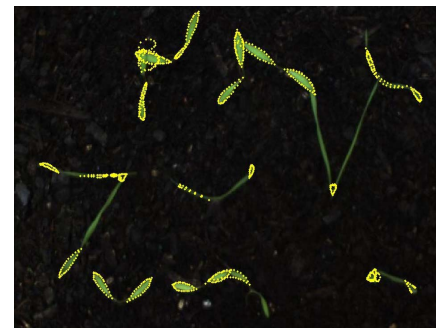

(e) deformable template result

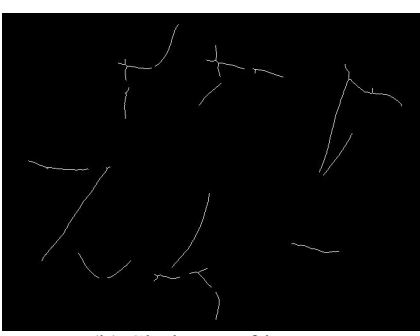

(b) Skeleton of leaves

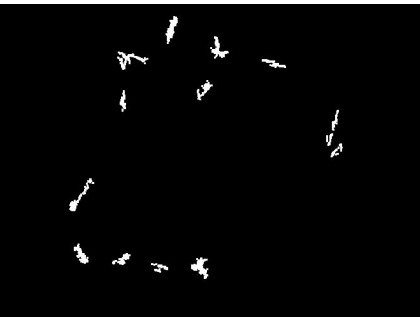

(d) Color based Bidens detection

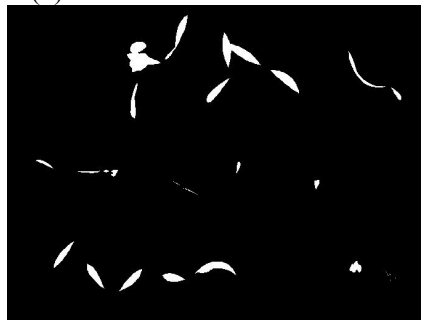

(f) Template enclosed area

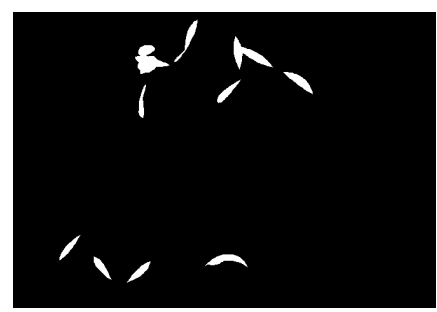

(g) Bidens map after shape validation Figure 9: Deformable template based shape validation

\section{EXPERIMENTAL RESUJLTS}

Bidens and wheat plants were grown on trays under laboratory conditions. A color camera with resolution of $1024 \times 768$ was used to capture images looking from the top with natural lighting.

Figure 10 shows the color based Bidens classification followed by deformable template based shape validation results. Table IV shows the accuracy of the algorithm.

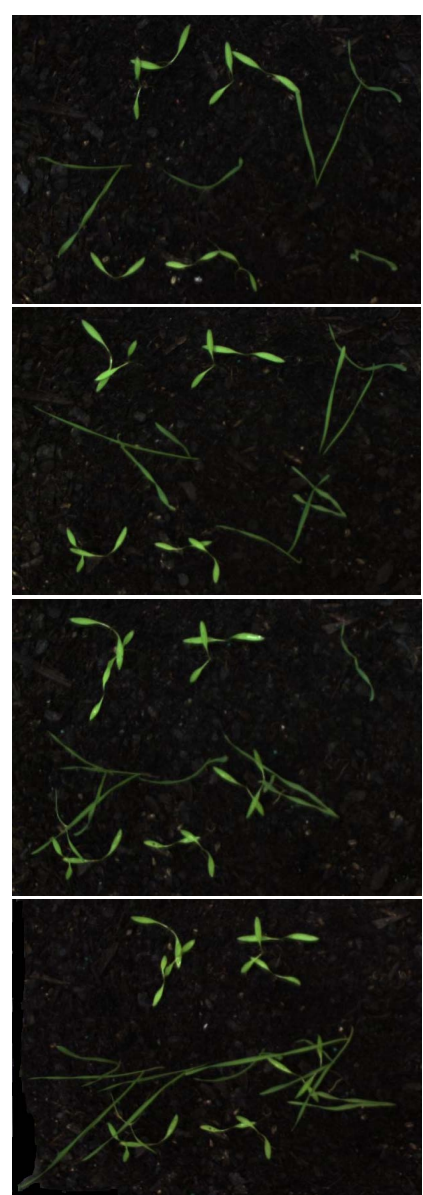

(a) Raw color images
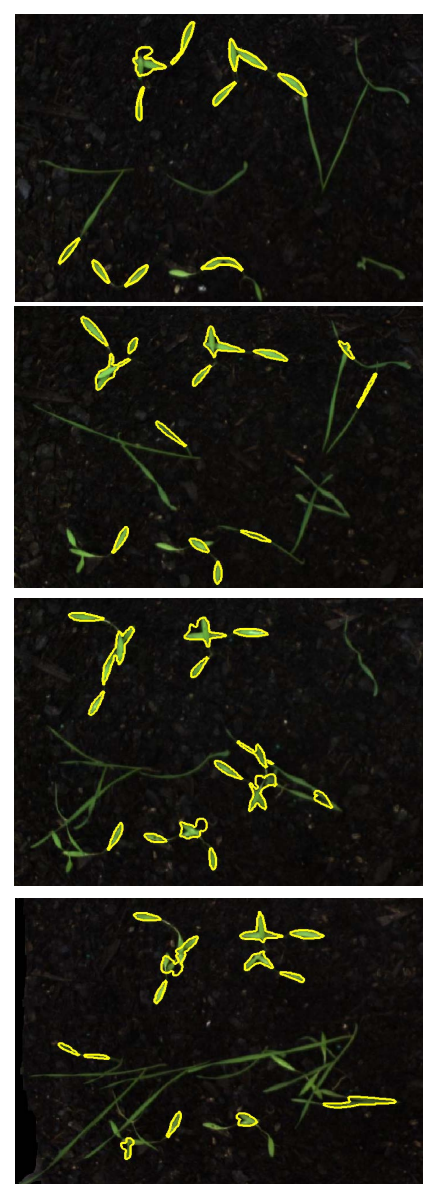

(b) Bidens detection results
TABLE IV: Color and shape validation results

\begin{tabular}{|c|c|}
\hline Detection rate & $80.0 \%$ \\
\hline False alarm rate & $9.9 \%$ \\
\hline
\end{tabular}


When comparing Table III with Table IV, it could be noted that the shape based validation contributed to $72 \%(35.6 \%$ to $9.9 \%$ ) reduction in false alarms. However, it also affected the detection rate of Bidens. The Bidens detection rate was reduced by $12 \%(90.7 \%$ to $80 \%)$. As our main target of this research is to destroy Bidens causing minimal damage to wheat it is utmost important to keep the false alarms rate to be low. Slight reduction in detection rates does not significantly affect the final outcome. This is mainly because of the fact that the platform with the camera is moving while capturing images at a fast rate with possible image overlaps.

Reason for reduced detection rate is attributed to strong overlapping of plant leaves. As shown in Figure 11, depending on the complexity of the scene, the deformable template based shape validation performance varies. Figure 11(a) shows high level of leaves overlapping which leads to missing initializations of templates. Figure 11(b) shows the problem of awkwardly grown deformable templates. Besides the overlapping problem, twisted wheat leaves being appeared as Bidens leaves in some cases leading to another cause of failure.

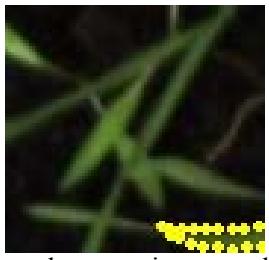

(a) Template growing error due to overlapping leaves

Figure 11: Possible cause of detection failure

\section{CONCLUSIONS}

In this paper, we have proposed a methodology for detecting Bidens in wheat farms. First, spectral analysis of Bidens and wheat leaves were performed. Based on spectral properties of leaves, it could be seen that visual color range of the spectrum is reasonably good for discrimination. Then a color based classification was presented with both high detection and false alarm rates. As the ultimate plan is to destroy the detected Bidens in wheat farms, high false alarm rates are not suitable for this application. High false alarms contribute to destroying wheat species. Therefore, we have introduced a shape based validation step to improve the false alarm rates. Shape based validation was nontrivial due to the presence of occlusions, which lead to reduction in detection rates. However, slight reduction in detection rates per image is acceptable especially with fast image rates while the camera is moving slowly.

As future work, we are in the process of investigating on improved shape validation techniques. We are also in the process of incorporating other sensor modalities such as NIR.

\section{ACKNOWLEDGMENT}

This work is supported by the ARC Centre of Excellence program, funded by the Australian Research Council (ARC) and the New South Wales State Government. The authors like to thank Dr Jay Katupitiya and Mr. Kim Son Dang for providing a picture of CASPA weeder and its description.

\section{REFERENCES}

[1] Pedersen, S. M., Fountas, S., Have, H., and Blackmore, B. S., "Agricultural robots-systems analysis and economic feasibility," Precision Agriculture. London, vol.7, pp. 295-308, 2006.

[2] Blackmore, S., Fountas, S., Tang, L., and Have, H., "Design specifications for a small autonomous tractor with behavioural control," $J$ Int Comm Agric Eng. 2004.

[3] Gerhards, R. and Oebel, H., "Practical experiences with a system for sitespecific weed control in arable crops using real-time image analysis and gps-controlled patch system," Weed Research. Vol.46, pp. 185-193, 2006.

[4] Gee, G., Bossu, J., Jones, G., and Truchetet, F., "Crop/weed discrimination in per-spective agronomic images," Computers and Electronics in Agriculture, vol60, pp. 49-59, 2008.

[5] Eaton, R., Katupitiya, J., Cole, A., and Meyer, C., "Architecture of an automated agricultural tractor: Hardware, software and control systems," Proceedings of the 2005 IFAC World Congress, 2005.

[6] Jurado-Exposito, M., Lopez-Granados, F., Stenciano, S., Garcia-Torres, L., and Gonzalez-Sndujar, J. L., "Discrimination of weed seedlings, wheat (triticum aestivum) stubble and sunflower (helianthus annuus) by near-infrared reflectance spectroscopy (nirs)," Crop Protection, vol22, pp. $1177-1180,2003$.

[7] Borregarrd, T., Nielsen, H., Norgarrd, L., and Have, H., "Crop-weed discrimination by line imaging spectroscopy," Journal of Agricultural Engineering Research, vo75, pp. 389-400, 2000.

[8] Gee, G., Bossu, J., Jones, G., and Truchetet, F., "Crop/weed discrimination in per-spective agronomic images," Computers and Electronics in Agriculture, vol60, pp. 49-59, 2008.

[8] Gee, G., Bossu, J., Jones, G., and Truchetet, F., "Crop/weed discrimination in per-spective agronomic images," Computers and Electronics in Agriculture, vol60, pp. 49-59, 2008.

[9] Eddy, P., Smith, A., Hill, B., Peddle, D., Coburn, C., and Blackshaw, R., "Architecture of an automated agricultural tractor: Hardware, software and control systems," Proceedings of Geoscience and Remote Sensing Symposium, 2006.

[10] Perzet, A. J., Lpez, F., Benlloch, J. V., and Christensen, S., "Colour and shape analysis techniques for weed detection in cereal fields," Computers and Electronics in Agriculture, vol25, pp. 197-212, 2000.

[11] Aitkenhead, M. J., Dalgetty, I. A., Mullins, C. E., McDonald, A. J. S., and Strachan, J. J. C., "Weed and crop discrimination using image analysis and artificial intelligence methods," Computers and Electronics in Agriculture, vol39, pp. 157-171, 2003.

[12] Hemming, J. and Rath, T., "Computer-vision-based weed identification under field conditions using controlled lighting," Journal of Agricultural Engineering Research, vol78, pp. 233-243, 2001.

[13] Holm, G. L., Plucknett, D. L., Pancho, J. V., and Herberger, J. P., "The World's Worst Weeds. Distribution and Biology," Krieger Pub Co., 2008

[14] OceanOptic (2008). Retrieved July 12, 2007, from http://www.oceanoptics.com.

[15] Weka 3: Data Mining Software in Java (2008). Retrieved July 12, 2007, from http://www.cs.waikato.ac.nz/ml/weka/.

[16] Zwiggelaar, R., "A review of spectral properties of plants and their potential use for crop/weed discrimination in row-crops," Crop Protection, vol17, pp. 189-206, 1998.

[17] Bradski, G., Kaehler, A., and Pisarevsky, V., "Learning based computer vision with intel's open source computer vision," Intel Technology Journal, vol9, pp. 119-130, 2005.

[18] Andrew Blake and Michael Isard, Active Contours, Springer-Verlag New York, Inc, 1998

[19] A.-G. Manh; G. Rabatel; L. Assemat; M.-J. Aldon, "Weed Leaf Image Segmentation by Deformable Templates," Journal of Agricultural Engineering Research, vol80, pp. 139-146, 2001. 